\title{
Editorial: Network Spread Models of Neurodegenerative Diseases
}

\author{
Ashish Raj ${ }^{1,2 *}$ and Yasser Iturria-Medina ${ }^{3}$ \\ ${ }^{1}$ Department of Radiology, Weill Cornell Medicine, New York, NY, United States, ${ }^{2}$ Department of Radiology, University of \\ California at San Francisco, San Francisco, CA, United States, ${ }^{3}$ Montreal Neurological Institute, McGill University, Montreal, \\ QC, Canada
}

Keywords: connectome, neurodegeneration, graph theory, Alzheimer's disease, protein aggregation, trans-neuronal transmission

\section{Editorial on the Research Topic}

\section{Network Spread Models of Neurodegenerative Diseases}

The emerging field of network neuroscience visualizes the brain as a graph consisting of nodes representing regions and edges as connections between them. This complex network supports efficient communication along neural projections, but also, unfortunately, the transmission and progression of neurodegenerative disorders like Alzheimer's disease (AD). If we could know the brain's network organization, could we then predict how degenerative processes might develop on this network? The answer is, increasingly, yes. This Research Topic collects a series of papers on various network models of dementia spread, focusing on disease-specific mathematical modeling rather than general graph theory.

\section{OPEN ACCESS}

Edited by:

Wendy Noble,

King's College London,

United Kingdom

Reviewed by:

Delphine Boche,

University of Southampton,

United Kingdom

*Correspondence:

Ashish Raj

asr2004@med.cornell.edu

Specialty section:

This article was submitted to

Neurodegeneration,

a section of the journal

Frontiers in Neurology

Received: 24 October 2018 Accepted: 14 December 2018

Published: 08 January 2019

Citation:

Raj A and Iturria-Medina Y (2019)

Editorial: Network Spread Models of

Neurodegenerative Diseases.

Front. Neurol. 9:1159.

doi: 10.3389/fneur.2018.01159

\section{MODELING NEURODEGENERATIVE DYNAMICS ON BRAIN NETWORKS}

Disturbances in global and local network organization are well documented in neurodegenerative diseases including AD (1), frontotemporal dementia (FTD) (2) and amyotrophic lateral sclerosis (ALS) (3). However, far more interesting than static networks is the potential to understand their dynamics-ongoing brain changes throughout disease (4). Broadly, this can happen in two ways: First, aberrant connectivity and network degeneration (5-7) via demyelination and axonal injury, secondary Wallerian degeneration, loss of signaling, axonal, and dendrite retraction. Second, disease factors can directly propagate along (possibly unchanging) neural connections, underpinned by "prion-like" protein aggregation followed by their trans-synaptic transmission (8-14). Thus, instead of being primarily impaired in degeneration, the network serves mainly as a conduit for disease transmission. Which type of dynamics predominates in neurodegeneration is a matter of lively debate.

In this Issue, Carbonell et al. provide a condensed historical review summarizing mathematical modeling of complex misfolded proteins mechanisms, both at the local/regional level and at the whole brain network level. They describe many recent network spread models, including models of cooperative spread (15), and communication cascades (16). In particular, an epidemic spreading model of network spread (17) was successfully validated on PET Amyloid- $\beta$ patterns in AD patients. An analytical Network Diffusion Model (NDM) mathematically derived the behavior of protein transmission as a graph heat equation under a connectivity-driven mechanism (18). This model is the basis of 2 papers in this Topic-Mezias and Raj and Pandya et al.

The accompanying paper by Mezias and Raj set out to apply the NDM, originally used on human data, to the problem of predicting the progression of $A \beta$ pathology in transgenic mouse models. They show that while NDM is capable of predicting $A \beta$ spread, it is not necessarily better than 
a model of spatial spread-one that does not involve network transmission. This is in contrast to the same authors' earlier publication showing a strong network mediation effect of tau spread (19), revealing a potentially important distinction between their respective mechanisms.

Pathological commonality and overlap is observed in various dementias: misfolded tau, A-beta and alpha-synuclein are present to varying degree in most degenerative diseases: AD, semantic dementia, FTD (20), ALS, dementia with Lewy bodies and posterior cortical atrophy (21). Pandya et al. explore this issue in a rare tauopathy called Progressive Supranuclear Palsy (PSP), which principally affects brainstem and striatal areas. Using the NDM Pandya et al. were able to recapitulate empirical PSP atrophy patterns. Although human imaging does not enable measurement of projection polarity, they presented a cross-species approach that transfers some directionality information from mouse to homologs regions in humans. Using this directional connectome, they found that anterograde and retrograde transmission give somewhat different spatiotemporal patterns of spread.

This raises important questions that can only be resolved by future advances in connectomics. Interestingly, the contributing paper by Neitzel et al. addresses this point directly in their perspective article. Using multimodal imaging data, including PET and functional MRI, they give a new perspective on how these techniques can be used to infer directionality of network connections in the human brain. As and when these techniques become increasingly refined and widely adopted, we predict that the issue of pathology spread on directional networks might assume critical ramifications.

The paper by Manuello et al. takes a meta-analysis approach for analyzing voxel-based morphometry data to understand the phenomenon of network spread. They report that in AD, gray matter alterations do not occur randomly across the brain but, on the contrary, follow identifiable patterns of distribution. This alteration pattern exhibits a network-like structure composed of co-altered areas that can be defined as co-atrophy network. The benefit of this type of data-driven approach is that it does not rely on specific hypotheses about network spread, unlike the papers by Mezias and Raj and Pandya et al.

A data-driven methodology was also adopted by Koval et al. which used the temporal alignment and combination of several short-term observation data to reconstruct the long-term atrophy

\section{REFERENCES}

1. Lo C-Y, Wang P-N, Chou K-H, Wang J, He Y, Lin C-P. Diffusion tensor tractography reveals abnormal topological organization in structural cortical networks in Alzheimer's disease. J Neurosci. (2010) 30:16876-85. doi: 10.1523/JNEUROSCI. 4136-10.2010

2. Kuceyeski A, Zhang Y, Raj A. Linking white matter integrity loss to associated cortical regions using structural connectivity information in Alzheimer's disease and fronto-temporal dementia: the Loss in Connectivity (LoCo) score. Neuroimage (2012) 61:1311-23. doi: 10.1016/j.neuroimage. 2012.03.039 history of $\mathrm{AD}$. This model provided a description of both the spatiotemporal patterns of cortical atrophy at the group level and the variability of these patterns at the individual level in terms of propagation pathways, speed of propagation, and age at propagation onset. Oxtoby et al. explored how the pathology propagates through the connectivity network via a data-driven event-based model. By analyzing the changes in the elderly brain's anatomical connectivity over the course of $\mathrm{AD}$, the authors clarified both the location and the sequence of changes to white matter connections. The results supported that degeneration of anatomical connectivity in the human brain may be an early and valuable biomarker when studying neurodegenerative diseases. Two other data-driven studies, Weber et al. and Huang et al., illustrate the methodological importance of considering focal axonal swelling and functional connectivity analysis for quantifiying memory deterioration rates in traumatic brain injury and evaluating clinical effects in infarction patients with dysphagia, respectively.

\section{SUMMARY AND OUTLOOK}

The present contributions encompass several cutting-edge techniques for investigating the phenomenon of networked spread in neurodegeneration, both model-based and data-driven. Together, they provide a framework for understanding the way the disease moves around within the brain network, that might adequately explain archetypal patterns of regional specificity in various dementias. Moving away from current statistical or descriptive graph theory, these papers trace underlying network dynamical processes. The emerging frontier of network spread modeling has the potential for wide applicability in diagnostics and therapeutic interventions.

\section{AUTHOR CONTRIBUTIONS}

All authors listed have made a substantial, direct and intellectual contribution to the work, and approved it for publication.

\section{ACKNOWLEDGMENTS}

AR acknowledges partial support by the following grants: R01 NS092802 and R01 EB022717 from the National Institutes of Health. 
7. Iturria-Medina Y. Anatomical brain networks on the prediction of abnormal brain states. Brain Connect. (2013) 3:1-21. doi: 10.1089/brain.2012.0122

8. Jucker M, Walker LC. Self-propagation of pathogenic protein aggregates in neurodegenerative diseases. Nature (2013) 501:45-51. doi: 10.1038 /nature12481

9. Frost B, Ollesch J, Wille H, Diamond MI. Conformational diversity of wildtype Tau fibrils specified by templated conformation change. J Biol Chem. (2009) 284:3546-51. doi: 10.1074/jbc.M805627200

10. Frost B, Diamond MI. Prion-like mechanisms in neurodegenerative diseases. Nat Rev Neurosci. (2010) 11:155-9. doi: 10.1038/nrn2786

11. Palop JJ, Chin J, Mucke L. A network dysfunction perspective on neurodegenerative diseases. Nature (2006) 443:768-73. doi: 10.1038/nature05289

12. Clavaguera F, Bolmont T, Crowther RA, Abramowski D, Frank S, Probst A, et al. Transmission and spreading of tauopathy in transgenic mouse brain. Nat Cell Biol. (2009) 11:909-13. doi: 10.1038/ncb1901

13. Liu L, Drouet V, Wu JW, Witter MP, Small SA, Clelland C, et al. Transsynaptic spread of tau pathology in vivo. PLoS ONE (2012) 7:e31302. doi: 10.1371/journal.pone.0031302

14. Iba M, McBride JD, Guo JL, Zhang B, Trojanowski JQ, Lee VMY. Tau pathology spread in PS19 tau transgenic mice following locus coeruleus (LC) injections of synthetic tau fibrils is determined by the LC's afferent and efferent connections. Acta Neuropathol. (2015) 130:349-62. doi: 10.1007/s00401-015-1458-4

15. Avena-Koenigsberger A, Misic B, Sporns O. Communication dynamics in complex brain networks. Nat Rev Neurosci. (2017) 19:17-33. doi: 10.1038/nrn.2017.149

16. Mišić B, Betzel RF, Nematzadeh A, Goñi J, Griffa A, Hagmann P, et al. Cooperative and competitive spreading dynamics on the human connectome. Neuron (2015) 86:1518-29. doi: 10.1016/j.neuron.2015.05.035
17. Iturria-Medina Y, Sotero RC, Toussaint PJ, Evans AC. Epidemic spreading model to characterize misfolded proteins propagation in aging and associated neurodegenerative disorders Sporns O, editor. PLoS Comput. Biol. (2014) 10:e1003956. doi: 10.1371/journal.pcbi.1003956

18. Raj A, Kuceyeski A, Weiner M. A network diffusion model of disease progression in dementia. Neuron (2012) 73:1204-15. doi: 10.1016/j.neuron.2011.12.040

19. Mezias C, LoCastro E, Xia C, Raj A. Connectivity, not region-intrinsic properties, predicts regional vulnerability to progressive tau pathology in mouse models of disease. Acta Neuropathol Commun. (2017) 5:61. doi: 10.1186/s40478-017-0459-z

20. Pereira JMS, Williams GB, Acosta-Cabronero J, Pengas G, Spillantini MG, Xuereb JH, et al. Atrophy patterns in histologic vs. clinical groupings of frontotemporal lobar degeneration. Neurology (2009) 72:1653-60. doi: 10.1212/WNL.0b013e3181a $55 \mathrm{fa} 2$

21. Rabinovici GD, Jagust WJ. Amyloid imaging in aging and dementia: testing the amyloid hypothesis in vivo. Behav Neurol. (2009) 21:117-28. doi: $10.1155 / 2009 / 609839$

Conflict of Interest Statement: The authors declare that the research was conducted in the absence of any commercial or financial relationships that could be construed as a potential conflict of interest.

Copyright $(\odot 2019$ Raj and Iturria-Medina. This is an open-access article distributed under the terms of the Creative Commons Attribution License (CC BY). The use, distribution or reproduction in other forums is permitted, provided the original author(s) and the copyright owner(s) are credited and that the original publication in this journal is cited, in accordance with accepted academic practice. No use, distribution or reproduction is permitted which does not comply with these terms. 